\title{
Neighborhood-corrected interface discontinuity factors for multi-group pin-by-pin diffusion calculations for LWR
}

\author{
José J. Herrero Nuria García-Herranz Diana Cuervo Carol Ahnert
}

\begin{abstract}
A B S T R A C T
Performing three-dimensional pin-by-pin full core calculations based on an improved solution of the multi-group diffusion equation is an affordable option nowadays to compute accurate local safety parameters for light water reactors. Since a transport approximation is solved, appropriate correction factors, such as interface discontinuity factors, are required to nearly reproduce the fully heterogeneous transport solution.

Calculating exact pin-by-pin discontinuity factors requires the knowledge of the heterogeneous neutron flux distribution, which depends on the boundary conditions of the pin-cell as well as the local variables along the nuclear reactor operation. As a consequence, it is impractical to compute them for each possible configuration; however, inaccurate correction factors are one major source of error in core analysis when using multi-group diffusion theory.

An alternative to generate accurate pin-by-pin interface discontinuity factors is to build a functionalfitting that allows incorporating the environment dependence in the computed values. This paper suggests a methodology to consider the neighborhood effect based on the Analytic Coarse-Mesh Finite Difference method for the multi-group diffusion equation. It has been applied to both definitions of interface discontinuity factors, the one based on the Generalized Equivalence Theory and the one based on Black-Box Homogenization, and for different few energy groups structures.

Conclusions are drawn over the optimal functional-fitting and demonstrative results are obtained with the multi-group pin-by-pin diffusion code COBAYA3 for representative PWR configurations.
\end{abstract}

\section{Introduction}

High fidelity multidimensional analysis tools for LWR allowing an accurate prediction of local parameters are required for core design and safety assessment. Such tools should integrate neutronic, thermal-hydraulic and fuel performance phenomena in a multiphysics approach (Chauliac et al., 2011).

The so-called next generation methods, based on explicit fully heterogeneous transport-theory core calculations (Joo et al., 2004; Weber et al., 2007) are promising, although impractical to assess local safety parameters since solutions are computationally prohibitive and thermo-hydraulic coupling is still challenging.

Then, the most common option nowadays for three-dimensional core analysis is to apply nodal methods, which perform homogenization inside an assembly and use some pin power reconstruction technique after the global nodal calculation (Bahadir and Lindahl, 2006; Joo et al., 2009). An alternative being explored and also based on nodal methods, avoiding pin power reconstruction, includes embedded lattice transport calculations (Ivanov et al., 2008; Zhang et al., 2008).

Other option consists on applying direct pin-by-pin methods (Tatsumi and Yamamoto, 2002; Kozlowski and Lee, 2002; Jiménez et al., 2010; Grundmann and Mittag, 2011). Since homogenization and thermo-hydraulic coupling is performed at the level of the pincell, the accuracy of the pin-wise fission rates will be higher than that of the nodal methods, so that they are considered as better candidates to compute local safety parameters.

Pin-by-pin methods can be based on a low order transport method, e.g. $\mathrm{SP}_{3}$ approach, or an improved solution of the multigroup diffusion equation. In both cases, it is well known that correction factors must be introduced in order to nearly reproduce the solution that would be obtained with a transport method. The Generalized Equivalence Theory (GET) (Smith, 1986) and the Superhomogenization method (SPH) (Hébert, 1993) are the most extended approaches. Besides, it is also possible to define different interface discontinuity factors (IDFs) than those of GET based on Black-Box Homogenization $(\mathrm{BBH})$ or Selengut normalization (Sánchez, 2009).

In any case, those correction factors should be calculated for the entire heterogeneous system if seeking to exactly reproduce the 
transport solution. However, that is impractical for whole core problems, and the required correction factors are usually pre-computed from pin-cell infinite lattice transport calculations. Since those parameters will be applied during the full core pin-by-pin diffusion calculation to core conditions for which they were not generated (the boundary conditions of any pin-cell in the core will differ from the zero-net-current condition), the diffusion solution will not be as accurate as that provided by the heterogeneous transport theory. This can be considered to be the major source of uncertainty in global core analysis when using multi-group diffusion theory.

Consequently, the correction factors obtained from infinite lattice calculations should be improved making them environment dependent, if the accuracy of the full core diffusion solution is to be increased.

Different attempts have been made in this sense. Environment corrected SPH factors were proposed by Yamamoto et al. (2004) and more recently, leakage dependent SPH factors were investigated by Takeda et al. (2008). Regarding GET interface discontinuity factors, a least-squares technique was used to generate a function that could approximate the real pin-cell IDFs using the homogeneous information available (Kozlowski, 2005). The implementation and testing in PARCS showed some numerical instability problems and further investigation was recommended.

Research to compute improved interface discontinuity factors has also been performed at the nodal level expanding the factors in terms of the surface current-to-flux ratio. The expansion coefficients are computed through the application of perturbation theory in the infinite-medium higher-order solution (Rahnema and McKinley, 2002). This method seems successful but, to our knowledge, it has not been applied at the pin level.

This paper suggests a possible correction of the pin-cell IDFs to consider the neighborhood effect based on the Analytic CoarseMesh Finite Difference (ACMFD) expressions for the multi-group diffusion equation (Chao, 1999), which have been further tested by our team for nodal core calculations (Aragonés et al., 2007).

The pin-cell IDFs in different condensed energy groups structures are computed and analyzed when changing the position of the pin inside the fuel assembly (and as a consequence the boundary conditions). Then, a functional-fitting based on terms involved in the ACMFD formulation is performed to incorporate the environment dependence. The coefficients of the functional-fitting can be determined, by applying a least-squares technique, from a few neighborhood cases compared to the high number of possible configurations to be encountered in a full core, thus reducing the amount of computational time needed to treat this effect if all the possible cases were to be considered.

The methodology has been implemented in the multi-group diffusion pin-by-pin code COBAYA3 (Herrero et al., 2007). Improved IDFs can be computed during fine-mesh diffusion calculations by correcting the infinite-lattice values as a function of the actual environment of the pin-cell in the core. Results obtained for different pin clusters representative of typical PWR assemblies are satisfactory.

In Section 2, the basic correction factors approaches to treat the errors associated to the use of the diffusion theory are presented, and the GET and BBH IDFs are chosen for this study. In Section 3, the IDF computed for different configurations are analyzed, and a functional-fitting to consider the environment effect is suggested. Numerical results are presented in Section 4 and finally, in Section 5 the main conclusions are summarized.

\section{Correction factor approaches}

Three possible correction factors have been considered for the present study: the ones from the SPH method, the classical GET interface discontinuity factors and the Black-Box Homogenization interface discontinuity factors.

\subsection{SPH factors}

SPH factors are defined to preserve both the reaction rates and the neutron streaming from one pin or node to its neighbors. To that aim, cross sections are corrected by a multiplicative factor $\mu$ which has to be obtained by an iterative procedure using the lower order formulation in the homogenized system and the following expressions:

$$
\begin{aligned}
& \tilde{\Sigma}=\mu \cdot \Sigma \\
& \tilde{\Sigma} \cdot \bar{\phi}^{\text {hom }}=\Sigma \cdot \bar{\phi}^{\text {het }} \\
& \mu=\frac{\phi^{\text {het }}}{\phi^{\text {hom }}}
\end{aligned}
$$

SPH factors are not direction dependent, but cell dependent. As a consequence, they cannot take into account the different heterogeneity and transport effects at each cell interface. Mixing the effects over all the interfaces in just one parameter implies a loss of information that makes the analysis of their environmental dependencies tougher. This is why the SPH method was not considered for the work performed in this paper.

\subsection{GET interface discontinuity factors}

GET interface discontinuity factors $f_{G}$ are defined to preserve the interface fluxes and net currents at the mesh interfaces from the transport to the diffusion solution. They are defined as the ratio of the heterogeneous interface flux $\phi_{s}^{\text {het }}$, the one coming from the transport solution; and the homogeneous interface flux $\phi_{s}^{\text {hom }}(2)$, which has to be consistent with the method used for the diffusion core calculation. When this method is the finemesh finite difference formulation, the discretization expression of the interface current results in Eq. (3), where $h$ is the mesh width.

$f_{G}=\frac{\phi_{s}^{\text {het }}}{\phi_{s}^{\text {hom }}}$

$J^{\text {het }}=-D \cdot \frac{\phi_{s}^{\text {het }} / f_{G}-\bar{\phi}^{\text {het }}}{h / 2}$

As these factors are interface dependent, they contain information about the orientation and position of the cell inside its environment. For instance, they are able to reflect the effect of a rotation of the environment around the cell, while SPH method is not able to isolate that effect. So they will facilitate the investigation of a functional-fitting depending on neighborhood.

In the particular case of a pin-cell in infinite lattice, the homogeneous interface flux is equal to the homogeneous average flux, which by definition will coincide with the heterogeneous one if the reaction rates are to be preserved; therefore we obtain the simple expression of the GET single-cell discontinuity factor $f_{0}$, equal at all the cell interfaces (4).

$J^{\text {het }}=0 \Rightarrow \phi_{s}^{\text {hom }}=\bar{\phi}^{\text {het }} \Rightarrow f_{0}=\frac{\phi_{s}^{\text {het }}}{\bar{\phi}^{\text {het }}}$

For the case where the pin is not in an infinite lattice, the discontinuity factors are different for each interface as the currents are, and they cannot be computed without obtaining the homogeneous interface flux corresponding to the lower order solution (5), e.g. diffusion.

$\phi_{s}^{\text {hom }}=\bar{\phi}^{\text {het }}-J^{\text {het }} \frac{h}{2 D}$ 


\section{3. $B B H$ interface discontinuity factors}

BBH interface discontinuity factors $f_{B}$ are defined to preserve the partial currents at the interfaces $J_{ \pm}^{\text {het }}$ from the transport to the diffusion solution (6). They enter inside the diffusion equation in the same way as the GET factors (3), so their use does not require further developments in the diffusion solver. They differ from the GET interface discontinuity factors, since the GET definition only preserves the partial currents if the higher order operator used to obtain the heterogeneous fluxes is also diffusion, while BBH factors preserve these partial currents for any higher order operator (Sánchez, 2009).

$f_{B}=2 \cdot \frac{J_{-}^{\text {het }}+J_{+}^{\text {het }}}{\phi_{s}^{\text {hom }}}$

In this work, the environment dependence of both GET and BBH interface discontinuity factors was analyzed.

\section{Environment dependence of the interface discontinuity factors}

\subsection{Configurations considered}

To perform this study, specifications for materials and geometries were taken from a NURESIM benchmarking document (Couyras, 2006) where four types of fuel pins, one guide tube pin and one control rod pin were defined, all with a common cell pitch of $1.26 \mathrm{~cm}$. The four types of fuel pins are a UOX $4.2 \mathrm{w} / \mathrm{o}$, the same UOX pin containing Gadolinium, a MOX pin with $5.2 \mathrm{w} / 0$ enrichment in Plutonium and another MOX pin with $7.8 \mathrm{w} / \mathrm{o}$ enrichment. The gap between fuel and cladding was not modeled. The control rod is a Silver, Indium and Cadmium (AIC) alloy.

All the transport calculations were performed with the NEWI code from the SCALE6.0 code package (Bowman, 2011) and its ENDF/B-7 library in 238 energy groups suitable for light water reactors. A subroutine was created to post-process the NEWT output file and get all the quantities in a more manageable format, including the computation of both GET and BBH IDFs. The capability of using a NEWT output file as a cross section library for COBAYA3 was also implemented, so the reference calculation could be reproduced by COBAYA3 with the exact interface discontinuity factors.

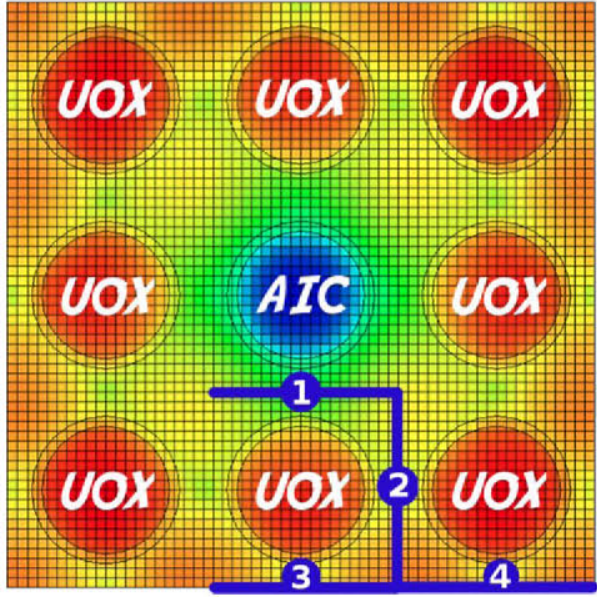

Fig. 1. Example of $3 \times 3$ cluster and interfaces adding information for UOX.

First, reference transport calculations with reflective boundary conditions and a critical buckling search were performed for all types of pins. Cross sections and interface values, including partial and net currents and fluxes, were obtained from NEWT in three different energy group structures (2, 4 and 8 ). The interface values were used to generate the interface discontinuity factors as defined in Eqs. (2) and (6).

Afterwards, different sets of $3 \times 3$ pin clusters with reflective boundary conditions were defined in order to perturb the partial currents at the interfaces of the pins from the zero-net-current condition. In some cluster configurations, the perturbing cell was placed only in the central position, as sketched in Fig. 1; due to the symmetries, only the four numbered interfaces yield new information about the interface discontinuity factors for the UOX cell. Note that interface number two produces information for the two cells to its left (2L) and right (2R). In other cluster configurations, the perturbing cells were also placed at other positions, so that different interface currents and transverse leakages for the four interfaces of each pin were obtained.

For instance, the UOX pin was perturbed with a water hole, a Gadolinium pin and a control rod; the Gadolinium pin was perturbed with a water hole, a UOX pin and a control rod. On the other hand, the MOX fuel pins were only perturbed with a water hole and a control rod, as Gadolinium is not present in such assemblies;

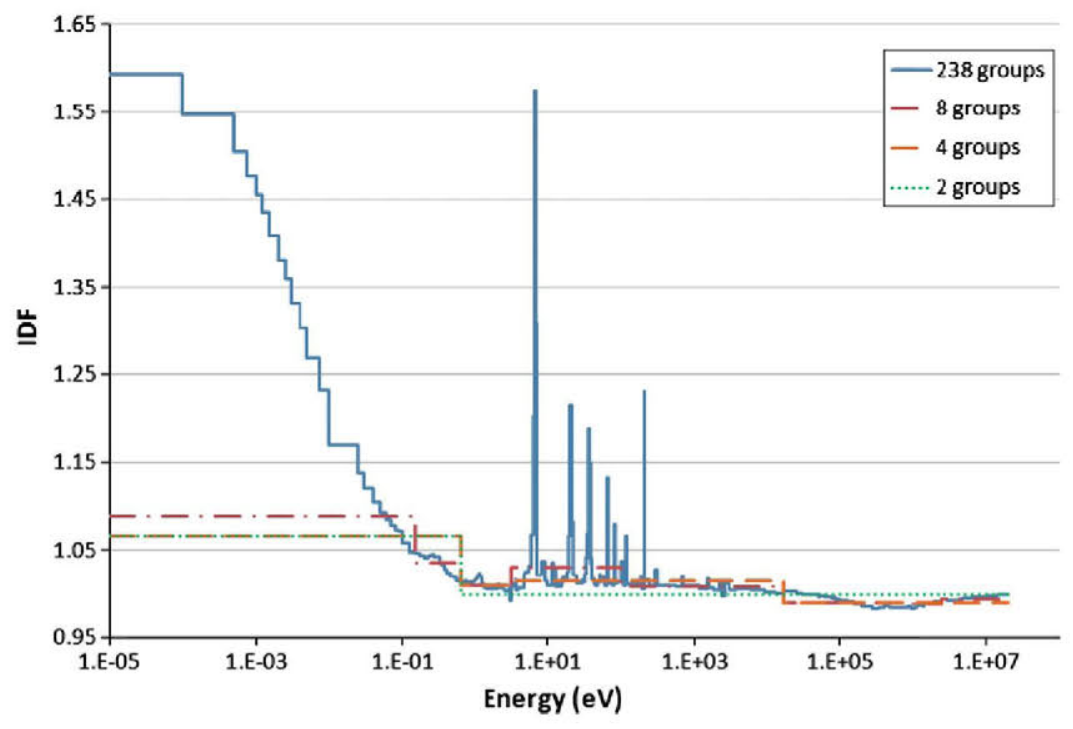

Fig. 2. GET interface discontinuity factors for the UOX single cell. 


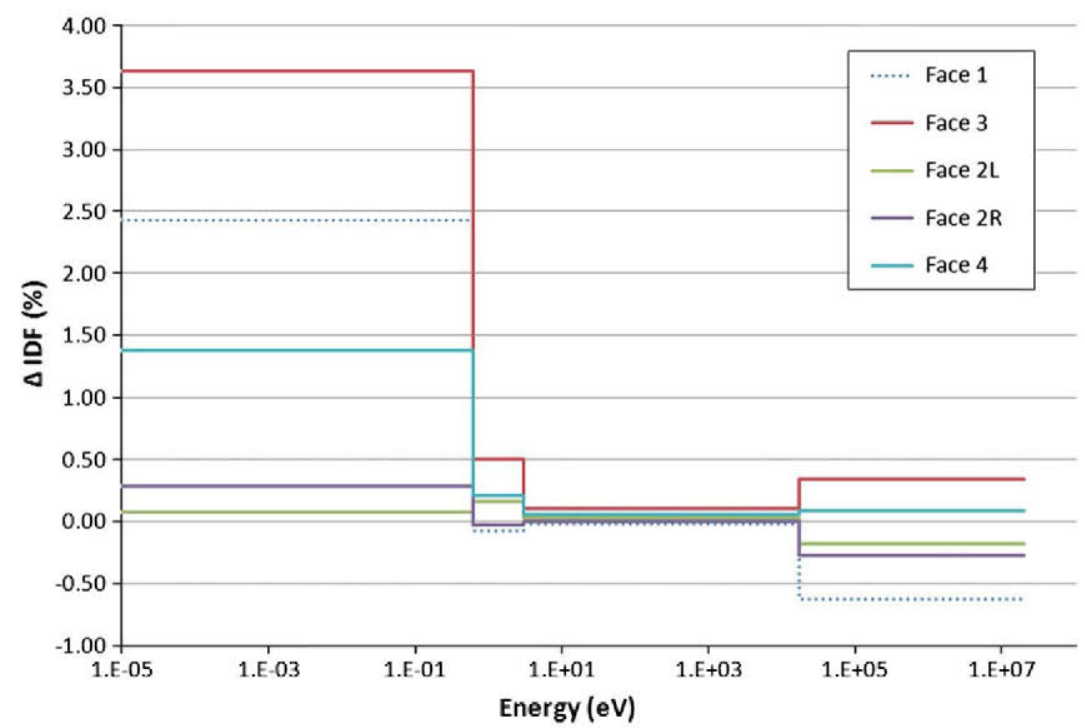

Fig. 3. GET IDF differences between a perturbed case and the single cell value.

uranium pins were also used to perturb the MOX clusters and vice versa, representing neighboring fuel assemblies.

\subsection{Analysis of the interface discontinuity factors behavior}

The pin-cell interface discontinuity factors computed for the defined configurations have been represented against different values influenced by the pin environment and available in the homogenized calculation. Let's first pay attention to the dependence of the IDFs with energy by representing their value for each energy group.

Fig. 2 shows the values of the GET factor for the UOX fuel pin in infinite-lattice (equal for all interfaces). From the 238 energy groups' representation it is clear that most of the correction is needed in the thermal range. Representations for few energy groups are in good agreement with the 238 groups profile above the thermal range while some loss of information in the thermal range can be noted. The profiles of the IDF for the BBH definition are quite similar to the ones of GET but with slightly different values; therefore we expect a close behavior of both parameterizations.

The IDFs for the $3 \times 3$ cluster represented in Fig. 1, where the UOX fuel pin boundaries are perturbed with a control rod in the central position, were computed. Fig. 3 shows the relative difference between those GET IDFs and the single cell value in 4 energy groups for each interface marked in Fig. 1.

That gives an idea of the correction level that should be introduced to consider the environmental effect on the infinite-lattice factors. It is lower than $10 \%$ for the configurations considered, although larger differences could be found for other configurations. It indicates that the nonlinear iteration required to compute the corrected IDF in the diffusion calculation should converge below $10 \%$ to result in an improvement of the solution.

\subsection{Proposed functional-fitting}

The ACMFD formulation for homogeneous nodes comes out explicitly from the analytic solution of the multi-group diffusion equations, with no approximation in 1D problems (Chao, 1999).

It relies on the transformation of the physical space of group fluxes into the modal space of the complete base of eigenvectors of the multi-group diffusion equation matrix. The resulting ACMFD coupling Eq. (7) are matrix-vector relations and, in this sense, they can be considered as a higher-order scheme with respect to the FMFD diffusion approximation, since it includes the effects of the intra-cell flux shape and the spectral variation.
$|\phi\rangle=A^{f}|\bar{\phi}\rangle-\frac{h}{2} A^{j} D^{-1}|J\rangle-\left(I-A^{f}\right) D^{-1} \lambda^{-1}|L\rangle$

In this equation, the quantities represented as $\mid$ kets $\rangle$ are vectors containing the value of the interface flux $|\phi\rangle$ and current $|J\rangle$, and the average cell flux $|\bar{\phi}\rangle$ for all energy groups; $A^{f}$ and $A^{j}$ are matrices affecting the fluxes and currents respectively coming from the analytical solution of each mode on each homogenous region; $|L\rangle$ is the transverse leakage term - arising from the generalization of the $1 \mathrm{D}$ ACMFD expression to more dimensions; $D$ stands for the diagonal diffusion coefficients matrix, $\lambda$ is the diagonal matrix of eigenvalues associated to each mode; and $h$ is the cell width.

If we consider the GET definition of the IDF and introduce the ACMFD expression to obtain a higher-order approximation of the heterogeneous interface flux, we get relation (8). In this equation, divisions are made component by component.

$\left|f_{G}\right\rangle=\frac{\left|\phi^{\text {het }}\right\rangle}{\left|\phi^{\text {hom }}\right\rangle}=\frac{A^{f}\left|\bar{\phi}^{\text {het }}\right\rangle-\frac{h}{2} A^{j} D^{-1}\left|J^{\text {het }}\right\rangle-\left(I-A^{f}\right) D^{-1} \lambda^{-1}\left|L^{\text {het }}\right\rangle}{\left|\phi^{\text {hom }}\right\rangle}$

Here, we can identify the first summand as the single-cell IDF when currents and transverse leakage equal zero, which would be obtained from the infinite lattice case $f_{0}$. While the rest of terms give an idea of what quantities would be suitable to take as neighborhood parameters, namely the heterogeneous interface current divided by the homogeneous interface flux and the heterogeneous transverse leakage divided by the homogeneous interface flux.

Let's focus on the homogeneous interface flux; it can be obtained by a simple relation from the finite difference approximation of the interface current (5). A different way around to get this value as a function of the cell buckling can be developed by expressing the total leakage from the cell as the summation of the interface currents and equating to the $\mathrm{DB}^{2}$ term (9). Introducing $q_{i}$ as the fraction of the current by the total leakage for each interface $i(10)$ and substituting the interface current with the homogeneous flux from (5), we can get Eq. (11), where the homogeneous interface flux is a function of the cell buckling.

$\sum_{\forall i} J_{i}^{\text {het }} \cdot h=D B^{2} \bar{\phi}^{\text {het }} h^{2}$

$q_{i}=J_{i}^{\text {het }} / \sum_{\forall i} J_{i}^{\text {het }}$ 


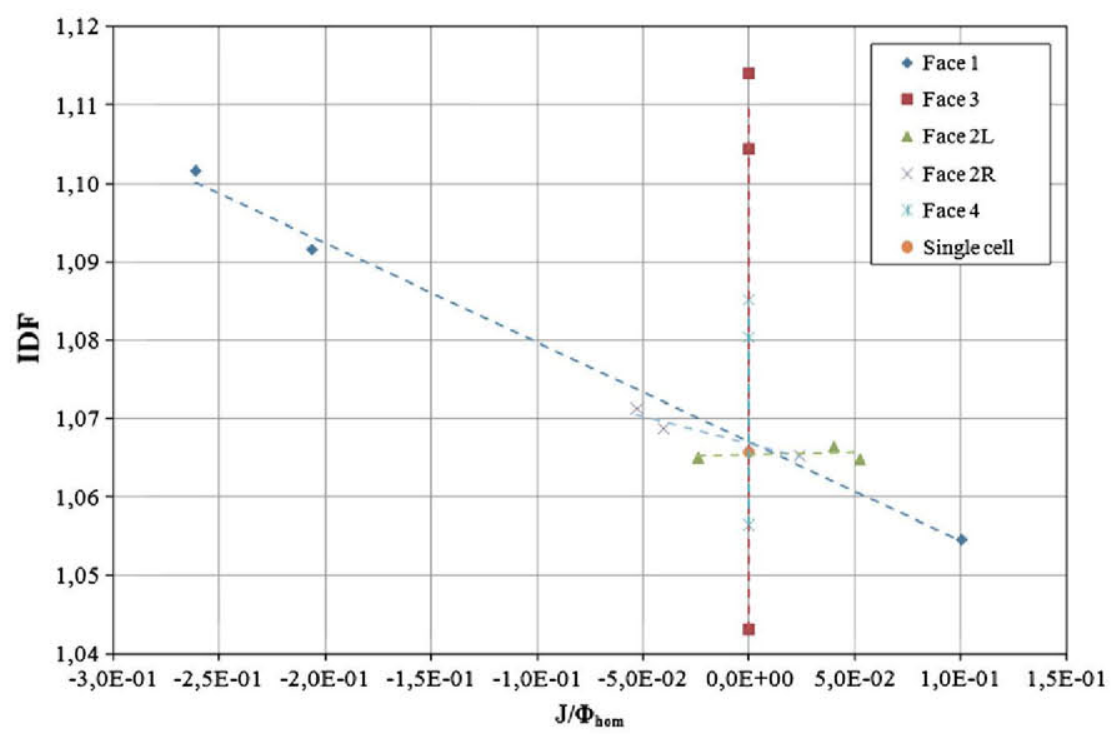

Fig. 4. GET IDF vs. interface current to homogeneous interface flux.

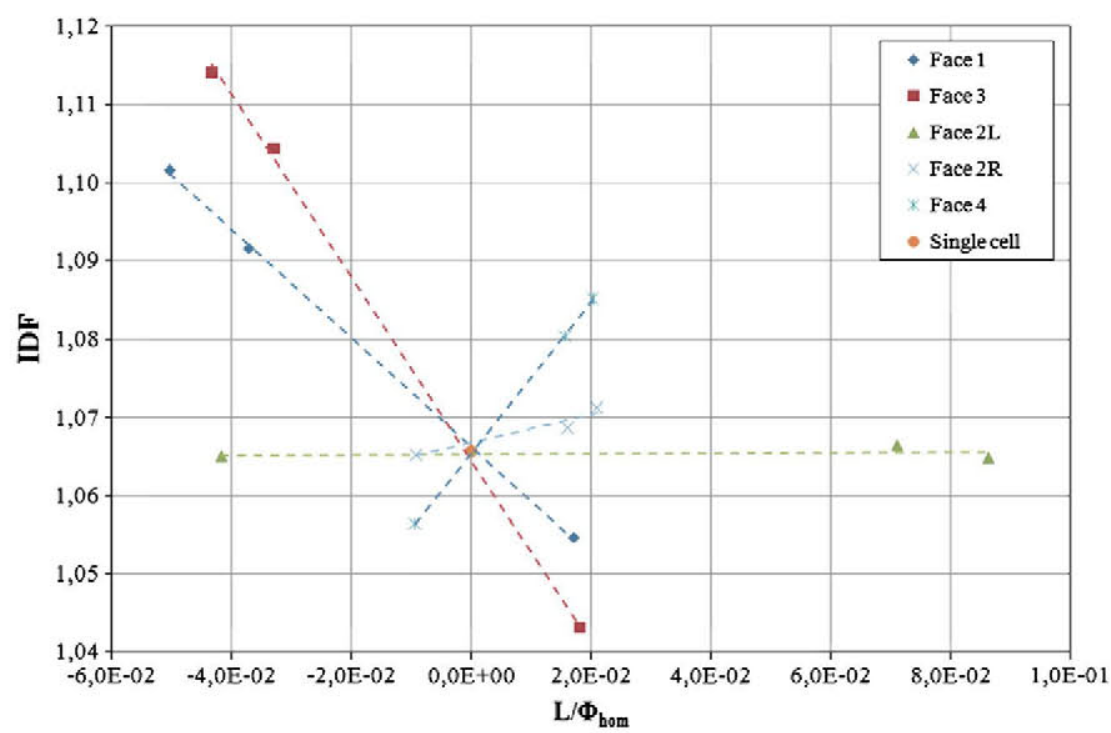

Fig. 5. GET IDF vs. transverse leakage to homogeneous interface flux.

$\phi_{s}^{\text {hom }}=\left(1-\frac{h^{2} q_{i}}{2} B^{2}\right) \bar{\phi}^{\text {het }}$

In the infinite lattice case the value of the homogeneous surface flux would be equal to the average heterogeneous cell flux. However, if the generation of the library has been made with critical $B_{1}$ search, the associated cell buckling is not zero and $q_{i}$ is equated to $1 / 4$ introducing a virtual current that reflects the effect of the critical buckling search on the IDF value. Therefore the IDF values from $k_{\text {eff }}$ and critical buckling search calculations are no longer equal. The buckling used in expression (11) must include the effect from the physical leakage in cases where the pin is surrounded by other types of cells, and the value of the buckling coming from the criticality $B_{1}$ search. Substituting (11) in (8) gives the following:

$$
\begin{aligned}
|f\rangle= & \frac{\left|\phi^{\text {het }}\right\rangle}{\left|\phi^{\text {hom }}\right\rangle}=\frac{A^{f}}{\left(1-\frac{h^{2} q_{i}}{2} B^{2}\right)}-\frac{\frac{h}{2} A^{j}}{\left(1-\frac{h^{2} q_{i}}{2} B^{2}\right)}\left|\frac{J^{\text {het }}}{D \bar{\phi}^{\text {het }}}\right\rangle \\
& -\frac{\left(I-A^{f}\right) \lambda^{-1}}{\left(1-\frac{h^{2} q_{i}}{2} B^{2}\right)}\left|\frac{L^{\text {het }}}{D \bar{\phi}^{\text {het }}}\right\rangle
\end{aligned}
$$

The dependence of each energy group on the rest of groups was neglected, thus changing the matrix coefficients to scalars and greatly simplifying the fitting process. Therefore the spectral effects due to neighborhood are not included in the formulation. In practice, those effects have being found to be of second order on importance.

The resulting expression seems to be not enough in all cases and also the cell buckling $\mathrm{B}^{2}$ was included for some energy groups and types of materials, which is justified in next section. Then, just four coefficients $m^{0}, m^{I}, m^{L}$ and $m^{B}$ per energy group need to be adjusted using Eq. (13); these coefficients are also environment dependent through the cross sections values but we take that influence as negligible.

$$
\begin{aligned}
f= & m^{0} \frac{1}{\left(1-\frac{h^{2} q_{i}}{2} B^{2}\right)}+m_{g}^{J} \frac{J^{h e t} / D}{\left(1-\frac{h^{2} q_{i}}{2} B^{2}\right) \bar{\phi}^{h e t}}+m_{g}^{L} \\
& \times \frac{L^{\text {het }} / D}{\left(1-\frac{h^{2} q_{i}}{2} B^{2}\right) \bar{\phi}^{\text {het }}}+m^{B} B^{2}
\end{aligned}
$$


This same type of parameterization has also been tested for the BBH IDF, although the derivation was made from the GET definition of the interface discontinuity factor. Consider that in the limit of using the diffusion operator also in the higher order solution, both IDF expressions will coincide given the relationship between interface partial currents, net current and flux in diffusion approximation.

\subsection{Physical interpretation}

As it has been derived in ( 8 ) from the ACMFD expression, it can be expected that the values of the IDF would depend on the heterogeneous average cell flux, on the heterogeneous interface current and on the transverse leakage, all of them divided by the homogeneous interface flux.

To understand the significance of each term, let us consider a critical buckling search calculation of a pin cell with zero-current boundary conditions. The flux curvature will evolve differently depending on the virtual leakage that makes the pin critical, and then, the IDF will change, being the same for all interfaces as the pin is symmetric.

This critical leakage is identified with the cell buckling introduced in (11) to substitute the ratio between the heterogeneous average cell flux and the homogeneous interface flux. Thus, the first dependence of the interface discontinuity factor comes from the fundamental flux shape inside the cell, and it is the main value defining the IDF. The rest of terms depending on the interfaces only modulate this main value.

The first modulating term is the interface current divided by the heterogeneous average cell flux, after substitution of the homogeneous interface flux by relationship (11). This ratio expresses the change in the flux curvature due to the change of the current value from the previous zero current condition.

To understand it better, let us consider that the virtual critical leakage is zero in the pin cell calculation and also assume that the pin is homogeneous for simplicity. Then, since there are no currents, the flux is flat. If an outbound current is introduced in one interface, the heterogeneous interface flux will be lower than the average flux, as the current is related to the flux gradient. The flux profile changes from flat to another curvature, what implies a change in the ratio of heterogeneous to homogeneous interface fluxes.
The second modulating term is related to the integrated leakage in the directions transversal to the interface considered. Its physical explanation has the same nature as the interface current dependency. It seems clear that an introduction of currents in an interface, different from the one where the IDF is being computed, has also an impact on the interface flux gradients of the rest of interfaces through a change at least in the average cell flux.

A third modulating term not coming from the ACMFD formulation and including the cell buckling was introduced in the proposed functional fitting (13). Its origin is related to the fact that the IDF actually depends on the pin homogenized cross sections. This can be seen in expression (12), where the elements of matrixes $A^{f}$ and $A^{j}$ are a combination of the homogenized cross sections values, which are in turn environment dependent. As there is evidence that this dependence of the cross sections can be parameterized with the buckling value of the pin (Cabellos et al., 1996), the IDF is also parameterized with the buckling to account for this effect.

\subsection{Advantages of including the corrected IDF in a pin-based library}

The final purpose of this methodology is to develop computational schemes for the generation of multi-group cross sections libraries with multifunctional dependence suitable for pin-by-pin transient calculations.

When creating a cross sections library, each kind of fuel pin, identified at least by its fresh isotopic composition and geometry, would correspond to a material type. As the interface discontinuity factors depend also on the position of the pin in the core, the number of types in the library should be much higher to take into account the position of the fuel pin inside the fuel assembly, as well as the position of the fuel assembly inside the reactor core.

In order to get an approximation to the number of material types to be considered for a fixed pin of a fuel assembly, let us focus on a classical $17 \times 17$ assembly. All the uranium fuel pins can be considered to be similar in material composition and geometry, but they are different when taking into account the surrounding cells, so that more than eight different positions of the same fuel pin can be found. Therefore a library with the IDFs explicitly stored

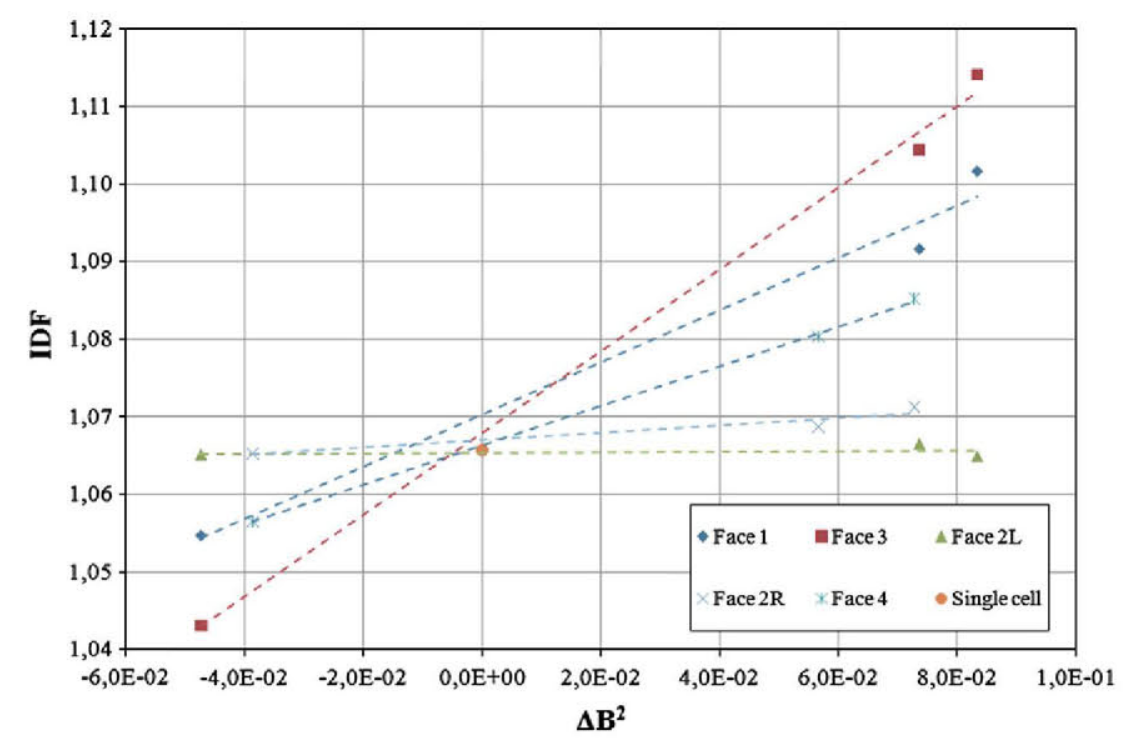

Fig. 6. GET IDF vs. the buckling change from the single cell case. 

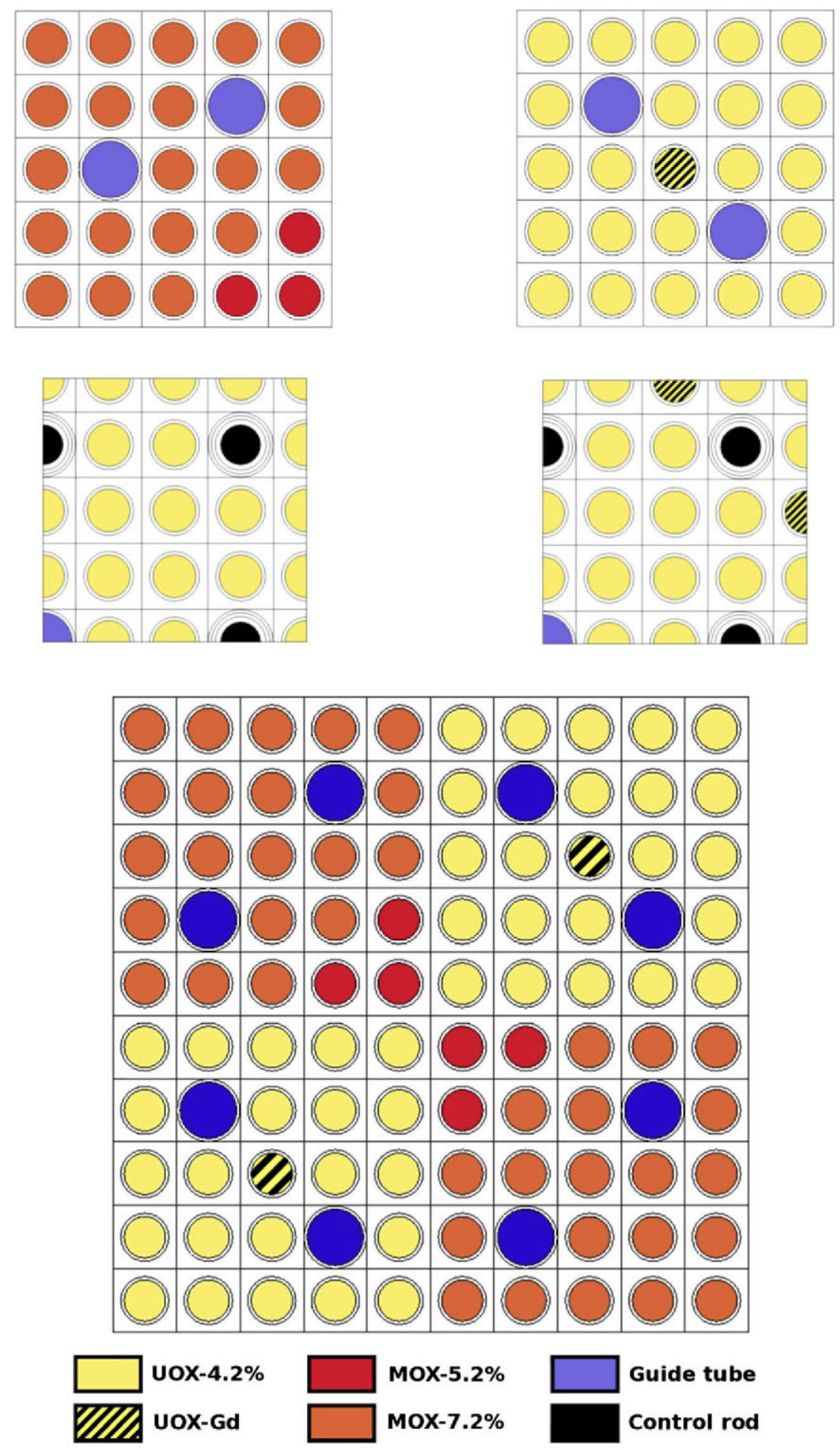

Fig. 7. Clusters defined to test the methodology.

for each interface would mean four interfaces, by eight different positions, by more than 10 possible situations of the fuel assembly inside the core. That would result in more than 320 values to be included in the library per combination of local variables.

As shown in Eq. (13), using the environment corrected IDF, only four factors are to be included in the library to cover the whole range of possible configurations. This simplification is at the additional cost of introducing a non-linear iteration loop in the convergence process for the IDFs whose convergence properties are presented in Section 4.2 .

\section{Numerical results}

\subsection{Results from the least-squares technique}

All the gathered data have been arranged in a single file including all the energy groups, interfaces and types of clusters considered. The code $R$ for statistics (R Development Core Team, 2011) has been used to treat these data and study the dependencies of the IDFs on interface values and buckling. In particular, the IDFs have been represented against those parameters to test for 
linearity on the dependencies. Prospection was made for different number of energy groups and for both definitions of the IDF, GET and $\mathrm{BBH}$.

Reasonably good agreement was encountered for all the energy groups considering the amount of simplifications done to get Eq. (13). Next figures show the IDF values for a UOX fuel pin when changing its neighborhood according to Fig. 1 . The represented values correspond to the thermal group in a four energy group structure.

In Fig. 4, the IDF is represented vs. the variation of the interface current to homogeneous flux. Trend lines are included for each interface numbered in Fig. 1, showing a linear dependence. One trend line was used per interface number since the mixture of effects is different for each interface. The buckling value was computed as the leakage needed by the cell to balance rest of terms in the diffusion equation divided by the average flux and the diffusion coefficient.

Fig. 5 shows the linear dependence of the IDF against interface transverse leakage to homogeneous flux and Fig. 6 against the cell buckling value.

The dependence with the cell buckling is not completely linear, remember that inclusion of the buckling did not come from the ACMFD formulation but from observation of the results and it is a good choice as represented in Fig. 6 . The buckling term gathers all the effects which are not included in the other two parameters, as the true expression which should be used for the heterogeneous flux is not the ACMFD expression for diffusion, but the one for transport (Chao, 2000) that has additional terms and cannot be simplified easily to be introduced inside a diffusion code.

The use of the buckling as an additional parameter is not always needed to get a good fitting. It has been used for the fuel pins and the guide tubes in the thermal energy groups, but not for the control rod.

\subsection{COBAYA3 results}

Five clusters were used to test the developed methodology (see Fig. 7), combining all the six types of pins listed in Section 3.1 with reflective boundary conditions:

- Cluster A is representative of a MOX fuel assembly including two guide tubes, three fuel pins containing Pu with a moderate enrichment, and rest of highly enriched Pu fuel pins.

- Cluster B is representative of a UOX fuel assembly including two guide tubes and one Gadolinium pin.

- Cluster C corresponds to a UOX fuel assembly with control rods inserted.

- Cluster D is equal to cluster $C$ but including Gadolinium pins to create a more challenging neighborhood problem.

- Cluster $\mathrm{E}$ is based on a combination of clusters $A$ and $B$ to test the methodology on the MOX and UOX assemblies interface.

All cluster calculations have been performed with NEWT and real values for the homogenized and collapsed cross sections and for the IDF per interface were produced. The use of such values in the pin-by-pin diffusion solver СOBAYA3 reproduced the results of the NEWT calculation in $k_{\text {eff }}$ and pin power distribution exactly in 2,4 and 8 energy groups, as expected.

The same calculations were performed with COBAYA3. First, without using IDFs; then, using the single-cell value computed by infinite lattice calculation; and finally, using the environment corrected discontinuity factors from GET and BBH definitions, which are different for each interface of the pin.

It should be noticed that the change in boundary conditions from the infinite lattice case will produce a modification in the
Table 1a

Eigenvalue deviations without IDF.

\begin{tabular}{lclc}
\hline $\boldsymbol{\Delta} \boldsymbol{k}_{\text {eff }}(\mathrm{pcm})$ & 2 Groups & 4 Groups & 8 Groups \\
\hline Cluster A & 22 & -129 & -164 \\
Cluster B & -302 & -341 & 209 \\
Cluster C & -257 & -434 & -262 \\
Cluster D & -765 & -827 & -378 \\
Cluster E & -166 & -229 & -56 \\
\hline
\end{tabular}

Table 1b

Maximum pin power error without IDF.

\begin{tabular}{llll}
\hline Relative error (\%) & 2 Groups & 4 Groups & 8 Groups \\
\hline Cluster A & 0.88 & 0.94 & 0.88 \\
Cluster B & 2.39 & 1.91 & 1.91 \\
Cluster C & 1.97 & 1.50 & 1.73 \\
Cluster D & 3.18 & 2.83 & 2.47 \\
Cluster E & 2.52 & 2.52 & 2.52 \\
\hline
\end{tabular}

Table 2a

Eigenvalue deviations using single-cell IDF.

\begin{tabular}{|c|c|c|c|c|c|c|}
\hline$\Delta k_{\text {eff }}(\mathrm{pcm})$ & 2g-GET & $2 \mathrm{~g}-\mathrm{BBH}$ & 4g-GET & $4 g-B B H$ & $8 \mathrm{~g}-\mathrm{GET}$ & $8 \mathrm{~g}-\mathrm{BBH}$ \\
\hline Cluster A & -48 & -20 & -50 & -74 & -58 & -90 \\
\hline Cluster B & 316 & 67 & 335 & 65 & 480 & 204 \\
\hline Cluster C & 350 & -236 & 433 & -355 & 631 & -209 \\
\hline Cluster D & 58 & -523 & 203 & -544 & 480 & -317 \\
\hline Cluster E & 283 & 140 & 314 & 121 & 528 & 323 \\
\hline
\end{tabular}

Table 2b

Maximum pin power errors using single-cell IDF.

\begin{tabular}{lllllll}
\hline Relative error (\%) & 2g-GET & 2g-BBH & 4g-GET & 4g-BBH & 8g-GET & 8g-BBH \\
\hline Cluster A & 1.39 & 0.97 & 1.39 & 0.90 & 1.52 & 1.01 \\
Cluster B & 0.60 & 0.60 & 0.60 & 0.60 & 0.60 & 0.96 \\
Cluster C & 2.08 & 1.97 & 1.73 & 1.39 & 1.85 & 1.62 \\
Cluster D & 1.71 & 2.12 & 1.24 & 1.77 & 1.03 & 1.77 \\
Cluster E & 3.74 & 2.49 & 3.66 & 2.34 & 3.98 & 2.65 \\
\hline
\end{tabular}

IDF; and also in the homogenized cross sections due to the change of the spatial flux distribution inside the cell, also called re-homogenization effect. The present study focuses only on the first effect, so that in all cases the cross sections coming from the real transport calculations were used, and there is no re-homogenization effect.

The deviations in $k_{\text {eff }}$ computed as the difference between the COBAYA3 result and the reference value from NEWT and the maximum relative pin power error for each cluster are presented the following tables; where red values refer to reactivity differences above $100 \mathrm{pcm}$ and pin power discrepancies above $1 \%$. A comprehensive study was made for sets of parameters in 2, 4 and 8 energy groups and for GET and BBH definitions with the aim of measuring the methodology performance in every situation.

Tables $1 \mathrm{a}$ and $1 \mathrm{~b}$ correspond to calculations without IDF. Results are, in general, not satisfactory for any cluster, regardless of the number of energy groups, so that the use of any kind of correction factors is mandatory.

The introduction of infinite-lattice interface discontinuity factors (see Tables 2a and 2b) slightly improves the pin power errors, but $k_{\text {eff }}$ prediction is, for some cases, even worse than the value obtained without correction factors. The relative difference between the infinite lattice value of the IDF and the one coming from the reference solution can be as high as a $30 \%$, which yields very inaccurate results. 
Table 3a

Eigenvalue deviations using an optimized parameterization.

\begin{tabular}{llrrrrr}
\hline$\Delta \boldsymbol{k}_{\text {eff }}(\mathrm{pcm})$ & $2 \mathrm{~g}-\mathrm{GET}$ & \multicolumn{1}{c}{ 2g-BBH } & \multicolumn{1}{c}{$4 \mathrm{~g}$-GET } & \multicolumn{1}{c}{$4 \mathrm{~g}-\mathrm{BBH}$} & $\mathbf{8 g - G E T}$ & $8 \mathrm{~g}-\mathrm{BBH}$ \\
\hline Cluster A & -16 & -9 & -4 & -16 & 0 & -25 \\
Cluster B & -51 & -39 & -67 & -35 & -90 & -58 \\
Cluster C & -50 & 18 & -86 & 71 & -153 & 27 \\
Cluster D & -26 & -148 & -108 & -43 & -109 & -49 \\
Cluster E & -76 & -68 & -23 & -29 & -1 & -2 \\
\hline
\end{tabular}

Table 3b

Maximum pin power errors using an optimized parameterization.

\begin{tabular}{lllllll}
\hline Relative error (\%) & 2g-GET & 2g-BBH & 4g-GET & 4g-BBH & 8g-GET & 8g-BBH \\
\hline Cluster A & $\mathbf{0 . 8 2}$ & $\mathbf{0 . 4 2}$ & $\mathbf{0 . 7 3}$ & $\mathbf{0 . 2 1}$ & $\mathbf{0 . 8 2}$ & $\mathbf{0}$ \\
Cluster B & $\mathbf{0 . 5 0}$ & $\mathbf{0 . 4 8}$ & $\mathbf{0 . 5 0}$ & $\mathbf{0 . 4 8}$ & $\mathbf{0 . 6 0}$ & $\mathbf{0 . 4 8}$ \\
Cluster C & 1.16 & 1.04 & $\mathbf{0 . 7 3}$ & $\mathbf{0 . 8 1}$ & 0.92 & $\mathbf{0 . 9 2}$ \\
Cluster D & 1.19 & 1.26 & $\mathbf{1 . 1 9}$ & $\mathbf{0 . 8 4}$ & 1.49 & 1.14 \\
Cluster E & 1.18 & $\mathbf{0 . 8 6}$ & $\mathbf{0 . 8 6}$ & $\mathbf{1 . 0 1}$ & $\mathbf{0 . 7 8}$ & $\mathbf{1 . 1 8}$ \\
\hline
\end{tabular}

Table 4

Maximum deviation of the parameterized IDF from exact values.

\begin{tabular}{lllllll}
\hline Relative error (\%) & 2g-GET & 2g-BBH & 4g-GET & 4g-BBH & 8g-GET & 8g-BBH \\
\hline Cluster A (\%) & 2.2 & 1.1 & 2.2 & 1.3 & 2.6 & 1.4 \\
Cluster B (\%) & 1.4 & 0.7 & 1.4 & 1.3 & 1.6 & 1.5 \\
Cluster C (\%) & 1.8 & 1.4 & 2.1 & 1.9 & 2.1 & 2.1 \\
Cluster D (\%) & 2.1 & 2.2 & 5.5 & 2.2 & 5.5 & 3.2 \\
Cluster E (\%) & 4.4 & 2.4 & 4.4 & 2.4 & 6.9 & 3.5 \\
\hline
\end{tabular}

An optimized parameterization was proposed and results are shown in Tables 3a and 3b. It can be seen a significant improvement of the accuracy in terms of $k_{\text {eff }}$ and pin powers for configurations $\mathrm{A}, \mathrm{B}$ and $\mathrm{C}$ for all energy groups. In the most challenging clusters $\mathrm{D}$ and $\mathrm{E}$, maximum errors are also reduced down below $1.5 \%$.

Notice that to get the analytical dependence, the matrix representation involved in the ACMFD formulation was disregarded. The fact of neglecting all the non-diagonal terms implies to discard the dependence of the discontinuity factors on the same parameters but coming from other energy groups. The reason to avoid the investigation of such approach is simply the impracticality of the method, as we should first compute a higher number of simplified $3 \times 3$ clusters to get enough points for a statistical adjustment of quality, increasing the computational demands. Then, a higher number of terms would have to be stored, reducing the advantages with respect to an explicit storage for each pin position. And last but not least, the non-linear character of the method would be stressed in a way that convergence of the IDF recomputation loop could be questionable.

The quality of the functional-fitting was measured for each cluster by computing the reference solution and feeding the exact values of the feedback parameters inside the optimized functional dependences. The computed IDF were then compared to the exact IDF, yielding errors shown in Table 4 . These values are always below $6 \%$, which means that the linear behavior hypothesis is quite valid on a wide range of variation of the IDF, even if the fitting equations are used under extrapolated conditions.

More important than the differences with respect to the transport solution is the fact that the IDF non-linear iteration must be converged to levels below the relative errors shown in the table.

The IDF iteration in COBAYA3 is performed outside of the $k$ eigenvalue loop, and it starts using the same IDFs on all interfaces $f_{0 g}$ to get a first result of the eigenvalue. The interface values and bucklings are then computed to get the new IDFs from expression (13), convergence of the IDFs is checked, and the computation proceeds with a new eigenvalue loop if necessary.
In order to achieve convergence of the IDF iteration, a damping on the calculated IDFs had to be used as high as 0.98 to avoid instabilities, meaning that the IDF value is updated with a $2 \%$ of the newly computed result and a $98 \%$ of the previous IDF for each iteration. With this damping value the number of recomputations of the IDF starting from the single cell value was always between 100 and 200 for a $1 \%$ level of convergence.

For each IDF loop, the calculation starts from a distribution closer to the next solution, so the computational time will be lower than for the initial iterations. Nevertheless, the computation time is still high for the solution of reactor cores where the source iteration for a fuel assembly can last minutes. In this sense, it would be necessary to improve the IDF loop combining it with the source loop or slightly relaxing the convergence level of the IDF; for instance, if changing the convergence criterion from $1 \%$ to $2 \%$, the loop ends approximately 20 iterations earlier.

Also the lowering of the damping factor from the current 0.98 file will alleviate convergence if possible, as it was chosen for maximum stability. As an example, using a damping factor of 0.85 was successful for all cluster computations except for the 8 energy groups solution using GET IDF. The number of iterations on the IDF was between 10 and 30 depending on the number of energy groups and the cluster type, which is quite acceptable.

\section{Summary and conclusions}

A new method to correct the multi-group pin-cell interface discontinuity factors as a function of the environment of the pin-cell in the core has been presented. Two different definitions for the interface discontinuity factors have been considered, Generalized Equivalence Theory and Black-Box Homogenization.

The proposed correction consists of building a functional-fitting of the interface discontinuity factors based on the analytical terms involved in the ACMFD expressions for the multi-group diffusion equation. The original expressions were simplified neglecting the cross terms between energy groups; and further expanded by including the cell buckling to compensate effects not considered in the ACMFD expression. That yields an optimized and simple relationship involving parameters available in the homogenized problem and suitable for pin-by-pin diffusion calculations.

The functional-fitting coefficients were computed from transport results by applying a least squares technique using the statistical code $R$. The developed functional expressions have been tested on representative clusters using the COBAYA3 pin-by-pin diffusion code.

Promising results in $k_{\text {eff }}$ and pin powers were obtained. In general, the performance of the environmental dependent BlackBox interface discontinuity factors was better compared to that of the GET factors. Further testing must be performed using problems involving a higher number of pins, like fuel assembly clusters, and with stronger heterogeneities (for instance introducing configurations with baffle and reflector). These problems will intensively test the convergence capability of the interpolation process which has a non-linear aspect that needs to be more deeply studied.

In summary, the developed methodology is able to catch the neighborhood effect in a very simple and practical way and is a good base for further developments.

\section{Acknowledgements}

This work is partially funded by the EC Commission under the 6th and 7th EURATOM Framework Programs, within the Integrated and Collaborative Project NURISP "Nuclear Reactor Integrated Simulation Project" under Contract 232124 (FI7O). It was also funded by the Spanish Science and Innovation Ministry within 
the FPU Program for teaching and researching formation under Grant AP2005-0667 for the first author.

\section{References}

Aragonés, J.M., Ahnert, C., et al., 2007. The analytic coarse-mesh finite difference method for multigroup and multidimensional diffusion calculations. Nucl. Sci. Eng. 157 (1), 1.

Bahadir, T., Lindahl, S.-Ö., 2006. SIMULATE-4 pin power calculations. In: Int. Conf. on the Physics of Reactors (PHYSOR 2006). Vancouver, Canada.

Bowman, S.M., 2011. SCALE 6: comprehensive nuclear safety analysis code system. Nucl. Technol. 174 (2), 126-148.

Cabellos, O., Aragonés, J.M., Ahnert, C., 1996. Generalized effects in two-group cross sections and discontinuity factors for PWRs. In: Int. Conf. on the Physics of Reactors (PHYSOR 1996). Mito, Japan.

Chao, Y.A., 1999. A theoretical analysis of the coarse mesh finite difference representation in advanced nodal methods. mathematics and computation. In: Reactor Physics and Environmental Analysis in Nuclear Applications (M\&C 1999). Madrid, Spain, pp. 117-126.

Chao, Y.A., 2000. Coarse mesh finite difference methods and applications. In: Proceedings of Reactor Physics and Mathematics and Computation into the Next. Millenium (PHYSOR-2000), 9404.

Chauliac, C., Aragonés, J.M., Bestion, D., Cacuci, D.G., Crouzet, N., Weiss, F.P., Zimmermann, M.A., 2011. NURESIM - a European simulation platform for nuclear reactor safety: multi-scale and multi-physics calculations, sensitivity and uncertainty analysis. Nucl. Eng. Des. 241 (9), 3416-3426.

Couyras, D., 2006. Specifications of the PWR NURESIM Core Physics Benchmarks. Part 1: Cell and Lattice Scope. EDF.

Grundmann, U., Mittag, S., 2011. Super-homogenisation factors in pinwise calculations by the reactor dynamics code DYN3D. Ann. Nucl. Energy 38 (10), 2111-2119.

Hébert, A., 1993. A consistent technique for the pin-by-pin homogenization of a pressurized water reactor assembly. Nucl. Sci. Eng. 113, 227-238.

Herreto, J.J., Ahnert, C., Aragonés, J.M., 2007. 3D whole core fine mesh multigroup diffusion calculations by domain decomposition through alternate dissections. In: Mathematics and Computations and Supercomputing in Nuclear Applications (M\&C/SNA-2007). Monterey, CA, USA, ISBN: 0-89448-059-6).
Ivanov, B., Müller, E., Ouisloumen, M., Ivanov, K., 2008. Embedded lattice transport calculations based on PARAGON-NEM code system for reactor core analysis. In: Int. Conf. on the Physics of Reactors (PHYSOR 2008). Interlaken, Switzerland.

Jimênez, J., Cuervo, D., Aragonés, J.M., 2010. A domain decomposition methodology for pin by pin coupled neutronic and thermal-hydraulic analyses in COBAYA3. Nucl. Eng. Des. 240 (2), 313.

Joo, H.G., Cho, J.Y., Kim, K.S. et al., 2004. Methods and performance of a threedimensional whole-core transport code DeCART. PHYSOR 2004. Chicago, IL, USA.

Joo, H.G., Yoon, J.I., Baek, S.G., 2009. Multigroup pin power reconstruction with twodimensional source expansion and corner flux discontinuity. Ann. Nucl. Energy $36(1), 85-97$.

Kozlowski, T., 2005. Spatial homogenization methods for pin-by-pin neutron transport calculations, thesis (Ph.D.). Purdue University, ISBN: 9780542349447.

Kozlowski, T., Lee, C. et al., 2002. Benchmarking of the multigroup, fine mesh, $\mathrm{SP}_{3}$ methods in PARCS with the VENUS-2 MOX critical experiments. PHYSOR 2002. Seoul, Korea.

R Development Core Team, 2011. R: A Language and Environment for Statistical Computing. Vienna, Austria, ISBN: 3-900051-07-0.

Rahnema, F., McKinley, M.S., 2002. High-order cross-section homogenization method. Ann. Nucl. Energy 29 (7), 875-899.

Sănchez, R., 2009. Assembly homogenization techniques for core calculations. Prog. Nucl. Energy $51(1), 14-31$

Smith, K.S., 1986. Assembly homogenization techniques for light water reactor analysis. Prog. Nucl. Energy 17 (3), 303.

Takeda, T., Kirimura, K., Fujita, Y., Yamaji, K., Sato, D., Matsumoto, H., 2008. Leakage dependent SPH factor for PWR whole core transport calculation. In: Int. Conf. on the Physics of Reactors (PHYSOR 2008). Interlaken, Switzerland.

Tatsumi, M., Yamamoto, A., 2002. Object-oriented three-dimensional fine-mesh transport calculation on parallel/distributed environments for advanced reactor core analyses. Nucl. Sci. Eng. 141 (3), 190-217.

Weber, D.P., Sofu, T., et al., 2007. High-fidelity light water reactor analysis with the numerical nuclear reactor. Nucl. Sci. Eng. 155 (3), 395-408.

Yamamoto, A., Tatsumi, M., Kitamura, Y., Yamane, Y., 2004. Improvement of the SPH method for pin-by-pin core calculations. J. Nucl. Sci. Technol. 41 (12), 1155

Zhang S., Tang C. Huang H., Chao Y.A., 2008. Feasibility of embedding nodal homogenization in next generation methods for 3D pin-by-pin core simulation. In: Int. Conf. on the Physics of Reactors (PHYSOR 2008). Interlaken, Switzerland. 